\title{
The Source of the Circulating Aggregate of Insulin in Type I Diabetic Patients Is Therapeutic Insulin
}

\author{
Maximo Maislos, Patricia M. Mead, Deborah H. Gaynor, and David C. Robbins \\ Metabolic Unit, Department of Medicine, University of Vermont, Burlington, Vermont 05405
}

\section{Abstract}

Circulating insulin immunoreactivity (IRI) in type I diabetic patients (insulin-dependent diabetes mellitus [IDDM]) includes a covalent aggregate about twice the size of insulin. These studies were designed to determine the source and conditions promoting the accumulation of this material.

Among 31 IDDMs, the aggregate made up $28 \pm 3.6 \%$ of the mean fasting plasma IRI. Five of these patients were restudied after $5 \mathrm{~d}$ of treatment with equidose intravenous insulin. The relative amount of the aggregate during subcutaneous treatment $(\mathbf{4 0} \pm \mathbf{8 . 0 \%})$ was indistinguishable $(P>0.7)$ from that at the termination of intravenous treatment $(41 \pm 6.8 \%)$.

To determine whether previous exposure to therapeutic insulin influenced the appearance and accumulation of the aggregate, we intravenously or subcutaneously infused insulin for 5 h in nine healthy volunteers (euglycemic clamp). At the termination of the high-dose intravenous infusion $\left(10 \mathrm{mU} \cdot \mathrm{kg}^{-1} \cdot \mathrm{min}^{-1}\right)$, the concentration of the aggregate was $81 \pm 18 \mu \mathrm{U} / \mathrm{ml}$, and it accounted for $2.9 \%$ of total IRI. At the conclusion of the other infusion protocols, the absolute amounts of aggregate were somewhat less, but they accounted for similar percentages. On polyacrylamide gel electrophoresis, the circulating aggregate was indistinguishable from a material of similar molecular weight contaminating commercial insulin.

We conclude that the insulin aggregate found in the blood of IDDMs originates in commercial insulin. Its appearance is independent of the route of insulin administration. Prolonged and continuous use of insulin may increase its concentration but is not necessary for its appearance. The potential biologic and immunologic consequences of the aggregate are important matters that need to be addressed.

\section{Introduction}

Insulin immunoreactivity in the blood of type I diabetic patients includes an aggregate of insulin that is approximately twice the size of monomeric insulin (1). The aggregate has physicochemical properties that clearly distinguish it from proinsulin of animal or endogenous origin. We detected this material in the blood of

A preliminary report of this work was presented in June, 1985, to the 45th Annual Scientific Meetings of the American Diabetes Association, Baltimore, MD.

Dr. Maislos' present address is Department of Medicine, Ben-Gurion University, School of Medicine, Beer-Sheva, Israel. Address reprint requests to Dr. Robbins. 1985

Received for publication 23 May 1985 and in revised form 25 October

J. Clin. Invest.

(C) The American Society for Clinical Investigation, Inc. 0021-9738/86/03/0717/07 \$1.00

Volume 77, March 1986, 717-723 each of twelve consecutively studied insulin-dependent diabetic individuals who took a variety of insulin preparations by either continuous subcutaneous insulin infusion (CSII) ${ }^{1}$ or conventional intermittent injection. The aggregate of insulin accounted for an average of $20 \%$ of the total circulating immunoreactive insulin (IRI).

Aggregates of insulin (thought to be covalent insulin dimers, references 2-4) account for a small percentage of all commercial insulin preparations $(3,4)$. Conditions such as temperature of storage and concentration of the insulin preparation are among those known to increase the amount of aggregate in the insulin bottle. On the other hand, aggregate formation may be accelerated by the injection of insulin into the subcutaneous tissues by virtue of increased temperature and an altered physicochemical environment.

The blood of diabetic patients chronically exposed to subcutaneous insulin contains an insulin aggregate of similar molecular weight to that contaminating commercial insulin. Therefore, it was our speculation that the source of these circulating aggregates was commercial insulin. In the present work, we enlarged our previous observation and studied the molecular weight composition of IRI in the blood of insulin-treated patients and normal volunteers infused with commercial insulin given by the subcutaneous or intravenous route. Our results strongly suggest that the source of the circulating insulin aggregate is indeed the therapeutic insulin. The subcutaneous tissues did not contribute to the formation of the insulin aggregate. Reduced biological activity of this high molecular weight insulin analogue may affect the action of monomeric insulin, account for discrepancies between the biological activity and immunologically measured concentrations of insulin, and have a role in the development of anti-insulin antibodies.

\section{Methods}

Study I: contribution of the insulin aggregate to total circulating IRI in patients with insulin-dependent diabetes mellitus (IDDM)

Subjects. 19 male and 12 female ketotic-prone diabetic patients (5), aged $19-48 \mathrm{yr}$, (mean $33 \pm 2.8 \mathrm{SEM}$ ), were recruited from the community and the University of Vermont Outpatient Clinic to donate blood. These patients had type I diabetes mellitus for a minimum of $5 \mathrm{yr}$, had no detectable plasma C-peptide response $(<0.03 \mathrm{pmol} / \mathrm{ml})$ to an intravenous glucagon challenge (6), and were without proteinuria or diminished creatinine clearance. The outpatients used a variety of insulin preparations as indicated in Table $\mathrm{I}$.

Procedures. $20 \mathrm{ml}$ of blood was withdrawn after an overnight fast and before the administration of the morning dose of insulin. The plasma was removed and stored at $-20^{\circ} \mathrm{C}$. Measurement of monomeric insulin and insulin aggregate was performed as described below.

1. Abbreviations used in this paper: CRC, Clinical Research Center, CSII, continuous subcutaneous insulin infusion; IDDM, insulin-dependent diabetes mellitus; IRI, immunoreactive insulin. 
Study II: crossover study: detection of the insulin aggregate in diabetic patients receiving continuous intravenous or subcutaneous insulin infusion

Subjects. Five participants of study I who used an open-loop infusion device to administer insulin volunteered to participate in this study. Four employed an AutoSyringe $\mathrm{AS}^{*} 6 \mathrm{C}$ infusion pump (AutoSyringe, Inc., Hookset, $\mathrm{NH}$ ) and the fifth used a Betatron-I device. During the 3 mo before study, treatment consisted of only beef-pork regular crystalline zinc insulin (Iletin-I U-100).

Procedures. The diabetic patients were admitted to the Clinical Research Center (CRC) at the University of Vermont for a period of $7 \mathrm{~d}$. During the 3 wk before admission the patients were instructed to avoid making any adjustments in their insulin doses. Throughout the prestudy period and during the study itself they consumed a diet based on the recommendations of the American Diabetes Association, and calories per day were calculated as $1.2 \times$ basal energy requirements (7). Physical activity during the CRC admission was restricted to walking about the research unit. On study day 1 and the morning of day 2 the subcutaneous insulin administration regimen was continued. Beginning at $0900 \mathrm{~h}$ on study day 2 , the indwelling subcutaneous insulin needle was removed and replaced with a 27 -gauge needle that was placed into a superficial arm vein. The same amount and type of insulin as had been used during the past $3 \mathrm{wk}$ was then delivered intravenously via the same infusion device. The pump was set to deliver the same basal infusion rate and premeal bolus as had been taken subcutaneously during the prestudy period. However, boluses were given 15 rather than 30 min before each meal to lessen the risks of hypoglycemia.

Needle placement and patency was tested at least every $12 \mathrm{~h}$ and the infusion set was replaced every $24 \mathrm{~h}$. Blood was obtained for analysis of IRI at the terminations of the subcutaneous and intravenous treatment phases. Samples were taken from the arm contralateral to that which was used for the insulin infusion. Attainment of strict normoglycemia was not an aim of this study. Thus, plasma glucose concentrations between 70 and $350 \mathrm{mg} / \mathrm{dl}$ were tolerated, and no alterations in insulin dose were required during the study period.

\section{Study III: appearance of the insulin aggregate in normal subjects during the intravenous or subcutaneous infusion of insulin}

Subjects. Nine healthy male volunteers (mean age $24.3 \pm 1.4 \mathrm{yr}$ ) without a family history of diabetes mellitus, who were within $10 \%$ of their ideal body weights (8), were recruited from the community to receive intravenous or subcutaneous insulin during euglycemic, insulin clamp studies (9). Criteria for participation included a normal assessment by physical examination, automated blood chemistries, complete blood count and 3-h oral glucose tolerance test (5).

Procedures. Each subject underwent both an intravenous and subcutaneous insulin infusion study, in random order, separated by at least $14 \mathrm{~d}$. The subjects were admitted to the CRC on the evening before the insulin clamp study. Five of the subjects received an insulin infusion of $1 \mathrm{mU} \cdot \mathrm{kg}^{-1} \cdot \mathrm{min}^{-1}$ during both the subcutaneous and intravenous infusion studies (low dose). The remaining four volunteers were given 10 $\mathrm{mU} \cdot \mathrm{kg}^{-1} \cdot \mathrm{min}^{-1}$ of insulin (high dose). Undiluted Iletin-I U-100 regular insulin was infused into either an arm vein or the subcutaneous tissue of the abdominal wall, at a point midway between the right superior iliac crest and the umbilicus. On the occasion of the high-dose studies, subcutaneous insulin was equally divided between two sites symmetrically placed along the left and right flanks as described immediately above. During each clamp, insulin was infused using a infusion pump (model M 355, Sage Instruments, Cambridge, MA), and delivered through a Micro-volume infusion set (AutoSyringe, Inc.) attached to a 1-ml tuberculin or 5-ml plastic syringe, depending on the rate of infusion. The durations of the intravenous and subcutaneous insulin infusions were 5 h. The infusion rate was doubled during the first $10 \mathrm{~min}$ (priming dose) in an attempt to more rapidly achieve steady state concentrations of serum insulin.
An 18-gauge catheter attached to sterile tubing was placed into an antecubital vein of the right arm for the servo-controlled infusion of 20 $50 \%$ (wt/vol) D-glucose. To obtain arterialized blood, a 19-gauge butterfly needle was placed in a retrograde manner in a right dorsal vein of the left hand, which was kept in a radiant heater at $70^{\circ} \mathrm{C}$. Blood sampling occurred at 5-min intervals for plasma glucose determination. In order to maintain plasma glucose at $\sim 90 \mathrm{mg} / \mathrm{dl}, 20$ or $50 \%$ glucose was administered by a variable rate infusion syringe pump (Harvard Apparatus Co. Inc., So. Natick, MA) based on a negative feedback algorithm (9).

Blood was obtained for measurement of serum insulin at 20-min intervals throughout the duration of the clamp study and during the $2 \mathrm{~h}$ after cessation of the insulin infusion. Plasma C-peptide (10), serum proinsulin-like material, and the higher molecular weight insulin aggregate were measured in samples obtained at 0 and 300 min, respectively.

The studies were approved by the Institutional Review Board on Human Experimentation, and informed consent was obtained from each volunteer before participation.

Control studies: in vitro incubation of insulin in whole blood, plasma, and buffer. To test the possibility that the insulin aggregate was formed in blood during laboratory processing, therapeutic insulin was added to whole blood, plasma, and buffer. After incubation, IRI was removed and analyzed for the presence of the insulin aggregate, as described below. Heparinized whole blood or plasma was obtained from fasting normal donors, and Iletin-I insulin was added to achieve a final concentration of $3,000 \mu \mathrm{U} / \mathrm{ml}$. A similar amount of insulin was added to $3 \mathrm{ml}$ of Tris$\mathrm{HCl}, \mathrm{pH}$ 7.6. Incubates were maintained at $37^{\circ} \mathrm{C}$ for $120 \mathrm{~min}$. At the end of the incubation, and after separation of plasma when necessary, the percentage of insulin aggregate in each tube was determined by gel filtration and insulin radioimmunoassay as described below.

\section{Analytical methods}

Sample processing and storage. Blood samples obtained from diabetic patients were immediately transferred to iced, plastic tubes containing an antiproteolytic and anticoagulant mixture (11), and processed according to the method of de Haën (12) to remove platelets.

Separation of IRI by molecular weight. $1 \mathrm{ml}$ of plasma or serum was placed over P-30 (fine) beads (Bio-Rad Laboratories, Richmond, CA). Plasma samples (from the diabetic patients) were processed through gel columns $(1.5 \times 90 \mathrm{~cm})$ in $3 \mathrm{M}$ acetic acid to separate insulin bound to anti-insulin antibodies. The same amount of insulin and insulin aggregate was detected in serum from the normal volunteers processed in either acetic acid or $0.1 \mathrm{M}$ Tris- $\mathrm{HCl}, \mathrm{pH} 7.6\left(20^{\circ} \mathrm{C}\right)$ columns $(1.0 \times 50 \mathrm{~cm})$. Thus, to minimize changes in buffers, serum from the normal volunteers was processed in the neutral columns. In both instances, the flow rates of the columns were 4-6 ml/h. IRI was measured directly in the samples processed in neutral buffer. In samples from the diabetic volunteers, acetic acid was removed by desiccation under vacuum at room temperature and the samples reconstituted in assay buffer before radioimmunoassay.

Polyacrylamide disc gel electrophoresis. High molecular weight insulin aggregate was removed from the plasma of diabetic volunteers by immunoaffinity chromatography and gel filtration as described in detail elsewhere (13). The relative mobility $\left(\boldsymbol{R}_{\boldsymbol{f}}\right)$ of $12 \mathrm{ng}$ of this material in $10 \%$ polyacrylamide gel ( $\mathrm{pH} 8.7$ ) was compared with that of the insulin aggregate (dimer) found in Iletin insulin.

Radioimmunoassay. Radioimmunoassay of insulin was accomplished by the method of Starr et al. (14), modified by the use of 8,000 -mol-wt polyethylene glycol to separate the bound from free insulin tracer. The assay employed the Wright antibody, and the lower limit of sensitivity was $2.5 \mu \mathrm{U} / \mathrm{ml}$. The interassay and intraassay coefficients of variation were 4.5 and $7.3 \%$, respectively. The assay was $\sim 70 \%$ less sensitive to intact human proinsulin than to insulin. A standard curve composed of highly purified porcine insulin dimer (kindly supplied by Dr. Ronald Chance, Eli Lilly \& Co., Indianapolis, IN) was parallel to the human insulin standards (15). Immunoreactive human C-peptide was measured by the method of Heding et al. (10) using goat antibody-I, kindly supplied by Dr. Mary Root (Eli Lilly \& Co.). 
Table I. Amounts of Insulin Aggregate and Total IRI

\begin{tabular}{clllc}
\hline Number of patients & Insulin type* $\ddagger$ & Mean dose & Total IRI & \multicolumn{1}{c}{ Ins Agg } \\
\hline & & $U / d$ & $\mu U / m l$ & $4 J / m l$ \\
16 & Beef-pork & $45(21-95)$ & $3134(53-13,580)$ & $470(12-1,365)$ \\
4 & Pork & $44(34-54)$ & $8134(2,145-13,370)$ & $1118(289-350)$ \\
11 & Human & $32(16-50)$ & $1890(14-8,700)$ & $162(2-350)$
\end{tabular}

Results are expressed as mean (range) values for each group. Ins Agg, insulin aggregate. * In the plasma of 31 IDDM patients grouped by type of insulin used. $¥$ Of the total group of 31 patients, 10 were treated with a CSII device; the rest used a combination of either neutral protein Hagedorn or lente and regular insulin.

Statistical methods. Results are expressed as mean \pm SEM. Comparisons were made using the paired $t$ test (two-tailed) or analysis of variance whenever appropriate. The minimum level of significance was 0.05 .

\section{Results}

Study I: contribution of the insulin aggregate to total circulating IRI in patients with IDDM

Despite lack of any detectable endogenous insulin or proinsulin synthesis (absence of C-peptide reactivity), gel filtration of serum from each of these 31 ambulatory diabetic patients consistently produced two peaks of IRI. Results of these analyses are summarized in Table I. One peak eluted in the position of insulin $(6,000 \mathrm{~mol} \mathrm{wt})$ and the other in the position of the proinsulin column marker. For the entire group, $28 \pm 3.6 \%$ (range $4-71 \%$ ) of the IRI had a molecular weight similar to proinsulin (between 9,000 and 12,000). The higher molecular weight insulin aggregate was neither proinsulin nor intermediates of proinsulin conversion, since it lacked, as we have also previously shown, C-peptide reactivity and resisted proteolytic (trypsin) conversion to a 6,000 mol-wt immunoreactive insulin-like substance, among other physicochemical properties clearly distinct from proinsulin of human or animal origin (1).

The insulin aggregate was present in the serum of every diabetic subject, regardless of the type or amount of insulin preparation used. Although the aggregate appeared to make up a larger average percentage of the total circulating IRI in patients using beef-pork insulin $(30.3 \pm 4.7 \%)$ than among those using highly purified pork $(14.2 \pm 5.4 \%)$ or biosynthetic human insulin $(29.7 \pm 7.1 \%){ }^{2}$ the differences were not significant. However, since the total amount of IRI was greatest among patients using the highly purified pork mixture, the absolute amounts of the aggregate $(1,118 \pm 696 \mu \mathrm{U} / \mathrm{ml})$ were also significantly $(P<0.03)$ greater than that observed in the blood of diabetic patients using beef-pork $(470 \pm 117 \mu \mathrm{U} / \mathrm{ml})$ or biosynthetic human insulin $(162 \pm 36 \mu \mathrm{U} / \mathrm{ml})$. Despite the relatively large range of total IRI in those using highly purified pork insulin, the mean total daily insulin dose taken by each group was not significantly different from one another $(P>0.1)$.

Study II: crossover study: detection of the insulin aggregate in diabetic patients receiving continuous intravenous or subcutaneous insulin infusion

To test the possibility that the environment of the subcutaneous tissue promoted the formation of the insulin aggregate, we mea-

2. The apparent discrepancy between percentage and absolute plasma insulin aggregate values (Table I) is due to the inclusion of patients with high plasma total IRI and low percentages of insulin aggregate and vice versa. sured the distribution by molecular weight of IRI in the blood of diabetic patients during subcutaneous and intravenous insulin treatment. From the group of 31 diabetic patients originally studied, five volunteered to participate. The results are summarized in Table II.

The absolute and relative amounts of high molecular weight insulin aggregate found at the termination of the intravenous period were indistinguishable from those occurring during subcutaneous treatment. The mean total concentrations of plasma IRI during the different treatments were not significantly different $(P>0.70$; Table II). Likewise, the mean percentages of the higher molecular weight insulin aggregate were indistinguishable $(40 \pm 8.0$ and $41 \pm 6.8 \%)$ at the termination of the subcutaneous and intravenous treatment periods, respectively.

\section{Study III: appearance of the insulin aggregate in normal} subjects during the intravenous or subcutaneous infusion of insulin

The data from previous studies were obtained from diabetic patients, and it was unclear whether the diabetic state was related in some way to the appearance of the aggregate. Therefore, we analyzed the blood of normal volunteers for the presence of the insulin aggregate during both the intravenous and subcutaneous infusions of therapeutic insulin.

High-dose subcutaneous insulin infusion $\left(10 \mathrm{mU} \cdot \mathrm{kg}^{-1}\right.$ - $\left.\min ^{-1}\right)$. During the procedure, endogenous insulin synthesis and release were monitored by the measurement of plasma C-peptide concentrations. As shown in Fig. 1, C-peptide levels fell by $44 \%$ from $540 \pm 178 \mathrm{pmol} /$ liter at $t=0 \mathrm{~min}$ to $305 \pm 71$ $\mathrm{pmol} / \mathrm{liter}$ at $t=300 \mathrm{~min} ; P<0.05$. Immediately before the study, basal mean concentration of the proinsulin-like material was $2.5 \pm 0.6 \mu \mathrm{U} / \mathrm{ml}$. At $t=300 \mathrm{~min}$, on termination of the insulin infusion, the absolute amount of immunoreactive material eluting from the column in this position (molecular weight 9,000-12,000; insulin aggregate) increased by a factor of 4.7 $(20 \pm 3 \mu \mathrm{U} / \mathrm{ml})$. At the termination of the infusion, the mean concentration of monomeric insulin had risen from basal values of $12 \pm 3.2$ to $504 \pm 34 \mu \mathrm{U} / \mathrm{ml}$.

High-dose intravenous insulin infusion $\left(10 \mathrm{mU} \cdot \mathrm{kg}^{-1} \cdot \mathrm{min}^{-1}\right)$. A qualitatively similar result was seen during the intravenous infusion of insulin. However, significantly greater absolute amounts of monomeric insulin and insulin aggregate were observed. The results of these studies are shown in Fig. 2. At the termination of the infusion, plasma C-peptide levels declined $65 \%$ (from $655 \pm 176$ to $292 \pm 70$ pmol/liter; $P<0.05$ ). Mean serum concentrations of monomeric insulin were $2,750 \pm 465$ $\mu \mathrm{U} / \mathrm{ml}$, levels $>5$ times that achieved during the equidose subcutaneous procedure. Likewise, the amount of the higher mo- 


\begin{tabular}{|c|c|c|c|c|c|}
\hline \multirow[b]{2}{*}{ Patient No. } & \multirow[b]{2}{*}{$\mathrm{U} / 24 \mathrm{~h}$} & \multicolumn{2}{|c|}{ Subcutaneous } & \multicolumn{2}{|l|}{ Intravenous } \\
\hline & & Total IRI & Aggregate & Total IRI & Aggregate \\
\hline & & $\mu U / m l$ & $\mu U / m l$ & $\mu U / m l$ & $\mu U / m l$ \\
\hline 1 & 50 & 287 & 177 & 377 & 200 \\
\hline 2 & 32 & 213 & 117 & 150 & 92 \\
\hline 3 & 72 & 2582 & 542 & 2256 & 613 \\
\hline 4 & 95 & 1015 & 342 & 1651 & 550 \\
\hline 5 & 39 & 58 & 16 & 40 & 12 \\
\hline Mean \pm SEM & $58 \pm 12$ & $831 \pm 467$ & $239 \pm 92$ & $895 \pm 445$ & $293 \pm 122$ \\
\hline
\end{tabular}

* Determination of total IRI and insulin aggregate in plasma of five IDDM patients studied during CSII and after $5 \mathrm{~d}$ of equidose insulin intravenous treatment with beef-pork crystalline zinc insulin.

lecular weight aggregate was considerably greater $(81 \pm 18 \mu \mathrm{U} /$ $\mathrm{ml}$ ). However, despite the significant differences in the absolute concentrations of monomeric insulin and insulin aggregate detected during the two procedures, the relative amounts of insulin aggregate (expressed as a percentage of the total IRI) were indistinguishable $(P>0.30)$. The higher molecular weight material accounted for a mean of $2.9 \pm 0.50$ and $3.8 \pm 0.43 \%$ of total IRI at the end of the high-dose intravenous and subcutaneous clamps, respectively.

Low-dose insulin infusion $\left(1 \mathrm{mU} \cdot \mathrm{kg}^{-1} \cdot \mathrm{min}^{-1}\right)$. To determine whether the insulin aggregate would be detected during the infusion of more physiologic amounts of insulin, similar analyses were made on samples obtained at the termination of low-dose intravenous and subcutaneous clamp studies. In comparison to prestudy basal levels, there was a twofold increase in the amount of 9,000-12,000-mol-wt material at the termination of the 5-h
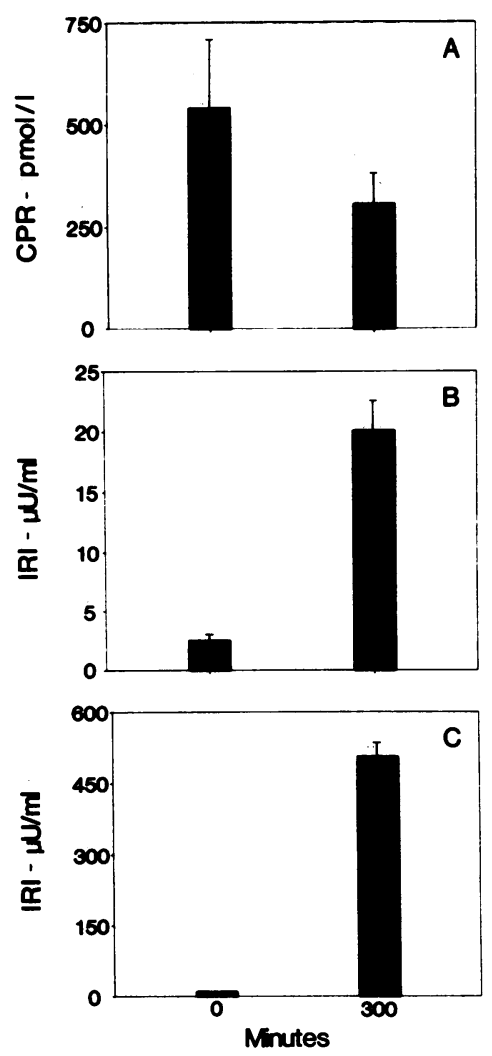

Distribution of IRI by molecular weight during subcutaneous insulin infusion in normal volunteers (mean of four studies). Shown are the concentrations of plasma C-peptide $(A)$, higher molecular weight insulin aggregate $(B)$, and monomeric insulin $(C)$ before $(0 \mathrm{~min})$ and at the end $(300 \mathrm{~min})$ of the insulin of beef-pork crystalline zinc insulin (U-100) at a rate of $10 \mathrm{mU} \cdot \mathrm{kg}^{-1} \cdot \mathrm{min}^{-1}$. procedure. At this time, the insulin aggregate accounted for $3.6 \pm 1.0$ and $4.8 \pm 0.6 \mu \mathrm{U} / \mathrm{ml}$ during the subcutaneous and intravenous studies, respectively. These values were significantly greater $(P<0.05)$ than the amount of proinsulin-like material measured at $t=0 \mathrm{~min}(1.8 \pm 0.7$ and $1.9 \pm 0.5 \mu \mathrm{U} / \mathrm{ml}$, respectively). These changes occurred despite a decrease in endogenous insulin secretion, as evidenced by a fall in plasma C-peptide ( $P$ $<0.05$ ) from basal levels of $528 \pm 157$ and $592 \pm 157 \mathrm{pmol} / \mathrm{liter}$ (at $t=0 \mathrm{~min}$ ) to $394 \pm 137$ and $376 \pm 125 \mathrm{pmol} / \mathrm{liter}$ (at $t=300$ min) during the subcutaneous and intravenous studies, respectively.

Characterization of the circulating insulin aggregate by gel filtration chromatography. The presence of the 9,000-12,000mol-wt insulin aggregate in the circulation raised the possibility, among others, that the material originated in the therapeutic insulin preparation. This hypothesis was strengthened by the
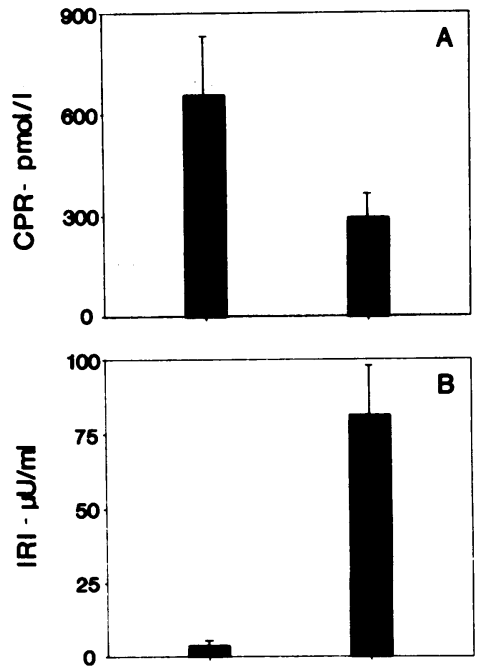

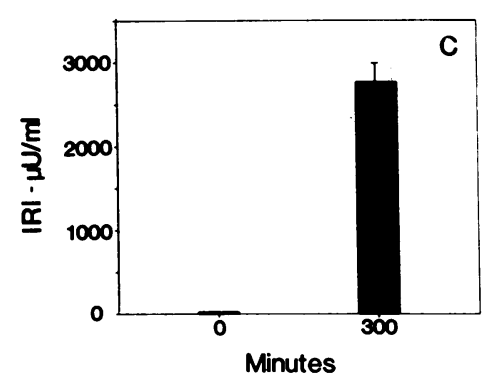

Figure 2. Distribution of IRI by molecular weight during intravenous insulin teers (mean of four studies). Shown are the concentrations of plasma C-peptide $(A)$, higher molecular weight insulin aggregate $(B)$, and monomeric insulin $(C)$ before $(0 \mathrm{~min})$ and at the end $(300 \mathrm{~min})$ of the infusion of beef-pork crystalline zinc insulin (U-100) at a rate of $10 \mathrm{mU} \cdot \mathrm{kg}^{-1} \cdot \mathrm{min}^{-1}$. infusion in normal volun- 
detection of a substance of similar molecular weight in each of the commercial preparations used routinely by these patients. Fig. 3 shows results typical of these analyses. High molecular weight material eluting in the position of the proinsulin marker was detected in each of the commercial insulin preparations of both domestic and European origins, and it typically accounted for $0.7-2.1 \%$ of the total as determined both by absorbance (276 $\mathrm{nm}$ ) or insulin radioimmunoassay (Fig. $3 \mathrm{~B}$ ). For comparison, this figure also shows the elution volumes (produced under identical conditions) of bovine insulin dimer (Fig. $3 C$ ) and IRI from the serum samples of a normal subject (Fig. $3 A$ ); it shows two peaks of IRI representing proinsulin-like material and insulin; and it shows the serum of a C-peptide-negative diabetic patient treated with beef-pork insulin (Fig. $3 \mathrm{D}$ ). Note that the insulin dimer (Fig. $3 \mathrm{C}$ ), although $50 \%$ larger than proinsulin, elutes under these conditions in the same position as the proinsulin marker.

Control studies: in vitro incubation of insulin in whole blood, plasma, and buffer. To test whether components of whole blood or the analytic and purification processes increased the concentration of insulin aggregate, an in vitro insulin incubation study was performed. Beef-pork insulin was added to heparinized whole blood, cell-free plasma, and buffer. After incubation at $37^{\circ} \mathrm{C}$, the insulin aggregate accounted for $1.5,1.3$, and $1.4 \%$ of total IRI in the incubates containing whole blood, plasma, and neutral buffer, respectively (results not shown). These amounts were less than the percentage of insulin aggregate $(2.1 \%)$ in the preparation of therapeutic insulin used for these experiments.

Physicochemical characterization of immunoaffinity-purified 9,000-12,000-mol-wt insulin aggregate: comparison to a contaminant in therapeutic insulin. The detection of the insulin aggregate in diabetic patients and normal volunteers during the
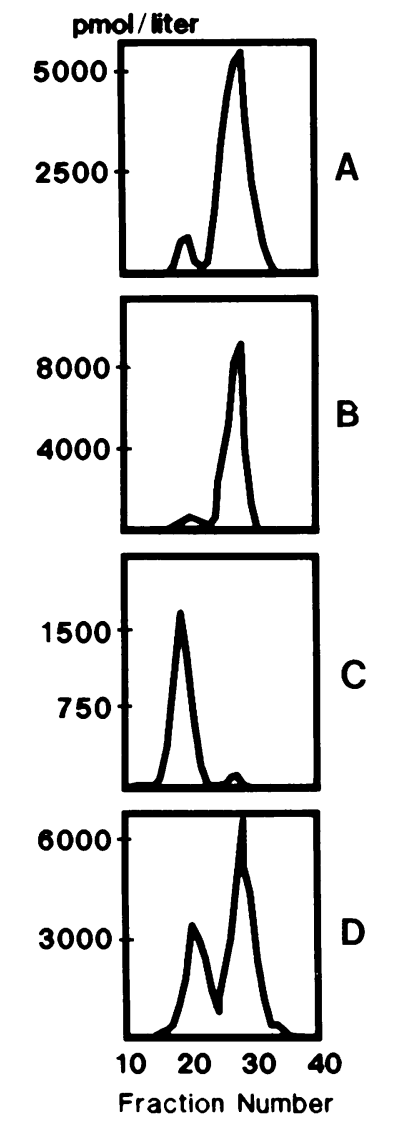

Figure 3. Representative samples of gel-filtered (P-30, Bio-Rad Laboratories $3 \mathrm{M}$ acetic acid, $0.005 \%$ bovine serum albumin) serum from a normal volunteer $(A)$, beef-pork regular crystalline zinc insulin $(B)$, bovine insulin dimer $(C)$, and plasma from a C-peptide negative diabetic patient treated with beef-pork insulin are shown. Fractions were assayed for immunoreactive insulin. The peak centered about fraction 20-25 represents proinsulin-like material $(A)$, or the higher molecular weight aggregate thought to be a covalent dimer of insulin $(B, C$, and $D$ ). therapeutic or experimental infusion of intravenous insulin strongly suggested that the higher molecular weight aggregate originated in the commercial preparation of insulin. To further explore this possibility, we applied the 12,000 -mol-wt material from therapeutic insulin (dimeric forms of insulin) and immunoaffinity-purified 9,000-12,000-mol-wt material from the blood of a type I diabetic patient to polyacrylamide gels and compared their mobilities. As can be seen in Fig. 4, the two materials produced similar heterogeneous patterns in the gels.

\section{Discussion}

In these studies we have attempted to define the conditions in which the aggregate of insulin arises, and to discover its source. Considering the fact that relatively high concentrations of insulin are injected into the subcutaneous tissues, it was possible that this environment contributed to the formation of the higher molecular weight material. However, our studies clearly showed that the appearance and accumulation of the material is independent of the route of insulin administration, since it can be found in the plasma of diabetic patients during intravenous insulin treatment. Furthermore, the insulin aggregate was found in the blood of normal volunteers after a relatively short period of intravenous or subcutaneous infusions of commercial insulin. Thus, the appearance of the material is dependent only on the delivery of commercial insulin.

The physicochemical similarities between the dimeric material in commercial insulin preparations and the material harvested from the blood of the diabetic patients added further weight to the conclusion that therapeutic insulin was the probable source of the insulin aggregate. However, since the amounts of the higher molecular weight material detected in the diabetic patients and normal volunteers were far greater than that in the parent insulin preparation, it is clear that the aggregate accumulates probably due to slower metabolic clearance relative to monomeric insulin. Further studies will be required to precisely define the pharmacokinetics and clearance mechanisms of the material.

It is noteworthy that there were both a considerably narrower range in amount and smaller total amounts of the circulating

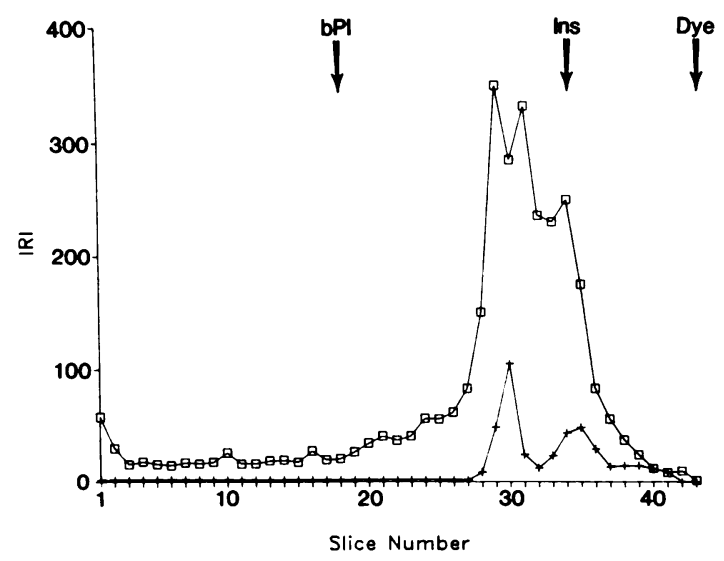

Figure 4. Polyacrylamide gel electrophoresis (10\%; pH 8.7) of immunoaffinity-purified, gel-filtered, higher molecular weight insulin aggregate from the serum of a diabetic patient $(\mathrm{X})$ and from an aliquot of the 12,000-mol-wt component of beef-pork crystalline zinc insulin U-100 ( $\square$ ). The arrows, from left to right, indicate the mobilities of intact beef proinsulin (bPI), porcine insulin (Ins), and the marker dye, analyzed in parallel gel columns. 
aggregate among the group of normal volunteers undergoing insulin infusion than among the diabetic patients. Continuous use of exogenous insulin and the presence of variable amounts of anti-insulin antibodies could facilitate the accumulation of the aggregate in the circulation of the diabetic patients. In addition, the anti-insulin antibodies would be likely to have differing affinities for monomeric insulin and the insulin aggregate, and this may explain the wide range of relative amounts of aggregate seen among patients taking similar doses of the same insulin preparation.

Although these investigations delineate the absence of any role of the subcutaneous tissues or diabetic state on the appearance of the aggregate, we are, as of yet, unable to offer a precise structural description of the aggregate itself. However, in this regard it should be emphasized that insulin is known to readily form a wide variety of high molecular weight substances including both covalent and noncovalent aggregates, zinc-associated hexamers, and macromolecular, fibrillar polymers (2-4, 16-22). Disulfide-bond rearrangement products have not been detected $(2,3)$. Among these known associations, covalent insulin aggregates (presumably dimers) are most likely to be the ones we have found in our patients. They have a similar molecular weight, the same mobility during electrophoresis, and they form spontaneously during storage of commercial insulin $(2,4)$. These interesting substances are formed by a covalent, isopeptide linkage. Apparently, the terminal amino acid of the insulin A-chain (A21 asparagine) is linked to either the $B 1$ phenylalanine (ABlinkage) or the $\mathrm{NH}_{2}$-terminal glycine (AA-linkage; reference 2). Such dimers account for $\sim 50 \%$ of the so-called b-component of crystalline insulin, a by-product of acid-alcohol recrystallization of pancreatic extracts $(2,3)$.

Although the vast majority of the b-component material is removed before packaging, a small amount either escapes purification or reforms in the packaged commercial insulin preparations. The relative amounts typically vary between 0.5 to $2.1 \%$ of the total hormone content among even the most purified forms of insulin available $(4,23)$.

The insulin aggregate found in the circulation of our diabetic patients and normal volunteers, and that in the therapeutic insulin preparations, share similar physicochemical characteristics. For instance, they both have the same elution volume on gel filtration. Both compounds are indistinguishable by gel electrophoresis and produce parallel displacement curves on dilution in the insulin radioimmunoassay (15). Preliminary separations performed by high performance reverse-phase liquid chromatography of the insulin aggregate recovered from the blood of diabetic patients have resulted in multiple peaks. These peaks elute under conditions and at retention times distinct from insulin, proinsulin, or intermediates of proinsulin conversion. The material recovered from commercial insulin preparations as well as dimeric standards behave similarly.

The fact that a large amount of circulating IRI in insulinusing diabetic patients is an aggregate, rather than monomeric insulin, raises several issues of interest and importance. Firstly, since dimeric forms of insulin apparently possess relatively little biologic activity $(2,15,24)$, these substances may act as partial antagonists or agonists to monomeric insulin, and produce a state of insulin resistance (15). Secondly, these compounds apparently have full or nearly full immunologic potential in the radioimmunoassay of insulin (15). Measurement of insulin concentrations in the plasma of insulin-using diabetic patients by radioimmunoassay may thus be misleading, since it includes compounds that differ markedly in their ability to promote insulin action. In our opinion, accurate measurement of IRI by radioimmunoassay in the blood of such individuals should be preceded by gel filtration. Without prior removal, the full immunologic potential of the insulin aggregate might contribute to the observed hyperinsulinemia of the euglycemic type I diabetic patient (25).

Finally, the insulin aggregate may have a role in the formation of antibodies to insulin, since it may be perceived as a foreign protein. Anti-insulin antibodies occur in diabetic patients treated solely with human insulin (26). Such preparations of either biosynthetic or semisynthetic origin contain the higher molecular weight material (26). In related studies, we have detected the aggregate in the circulation of diabetic patients treated with these materials (27).

In conclusion, our studies have demonstrated that the molecular species with insulin immunoreactivity in the blood of insulin-dependent patients includes an aggregate of insulin. It accounts for a variable but frequently significant proportion of total IRI. The well-known tendency of insulin to form covalent associations, and several physicochemical similarities between the material in commercial insulins and that in the blood, suggest that we have detected dimeric forms of insulin. Since the aggregate can be found in normal subjects during infusion with insulin, it is most probably of exogenous origin (the therapeutic insulin). Relatively higher percentages of the aggregate in the circulation than in the insulin bottle indicate that it is cleared more slowly than monomeric insulin. Binding to anti-insulin antibodies in the blood of the diabetic patients may further prolong its halflife and increase its concentration. The discrepancy between the biologic and immunologic activities of circulating insulin aggregate raises doubts regarding the specificity and accuracy of the insulin radioimmunoassay when it is used to measure insulin in the blood of insulin-treated diabetic patients or normal volunteers infused with insulin. The potential immunological and biological consequences of this material, its space of distribution, and mechanisms of clearance are important issues that need to be clarified in future investigations.

\section{Acknowledgments}

We thank Ms. Mary Chozie for her technical expertise.

Dr. Robbins is a recipient of a grant from the National Institutes of Health (NIH) (R01 AM 34101-01) and a grant-in-aid from the Eli Lilly \& Co. The Clinical Research Center at the University of Vermont is supported by the NIH (RR-109).

\section{References}

1. Robbins, D. C., S. Shoelson, P. M. Mead, H. S. Tager, and D. H. Gaynor. 1985. Products of therapeutic insulins in the blood of insulintreated type I diabetic patients. Diabetes. 34:510-519.

2. Helbig, H.-J. 1976. Insulindimere aus der b-Komponente von Insulin-präparationen. Ph.D. Thesis. Rheinisch-Westfälische Technische Hochschule Aachen. Aachen, Federal Republic of Germany.

3. Steiner, D. F., O. Hallund, A. Rubenstein, S. Cho, and C. Bayliss. 1968. Isolation and properties of proinsulin, intermediate forms, and other minor components of crystalline bovine insulin. Diabetes. 17:725736.

4. Brange, J., L. Langkjaer, S. Havelund, and E. Sorensen. 1983. Chemical stability of insulin: neutral insulin solutions. Diabetologia. 25: 143. (Abstr.)

5. National Diabetes Data Group. 1979. Classification and diagnosis 
of diabetes and other categories of glucose intolerance. Diabetes. 28 : 1039-1057.

6. Faber, O. K., and C. Binder. 1977. C-peptide response to glucagon: a test for the residual B-cell function in diabetes mellitus. Diabetes. 26: 605-610.

7. Harris, J. A., and F. G. Benedict. 1919. A Biometric Study of Basal Metabolism in Man. Carnegie Institute, Washington. Publication No. 279. J. B. Lippincott \& Co., Philadelphia.

8. Metropolitan Height and Weight Tables. 1983. Metropolitan Life Insurance Co., New York.

9. De Fronzo, R. A., J. D. Tobin, and R. Andres. 1979. Glucose clamp technique: a method for quantifying insulin secretion and resistance. Am. J. Physiol. 237(Endocrinol. Metab. Gastrointest. Physiol. 6): E214-E223.

10. Heding, L. G. 1975. Radioimmunological determination of human C-peptide in serum. Diabetologia. 11:541-548.

11. Fujikawa, K., M. E. Legaz, and E. W. Davie. 1972. Bovine factors $\mathrm{X}$ and X2 (Stuart factor). Isolation and characterization. Biochemistry. 11:4882-4891.

12. de Haën, C., S. A. Little, J. M. May, and R. H. Williams. 1978. Characterization of proinsulin-insulin intermediates in human plasma. J. Clin. Invest. 62:727-737.

13. Robbins, D. C., S. E. Shoelson, A. H. Rubenstein, and H. S. Tager. 1984. Familial hyperproinsulinemia: two cohorts secreting indistinguishable type II intermediates of proinsulin conversion. J. Clin. Invest. 73:714-719.

14. Starr, J. I., D. L. Horwitz, A. H. Rubenstein, and M. E. Mako. 1979. Insulin, proinsulin and C-peptide. In Methods of Hormone Radioimmunoassay. B. M. Jaffe and H. R. Behrman, editors. Academic Press, Inc., New York. 613-642.

15. Horton, E. S., M. Hirshman, L. Wardzala, and D. C. Robbins. 1985. Diminished in vitro biologic and binding activity of covalent insulin dimers: a possible explanation for decreased insulin action in IDDM. Diabetes. 34(Suppl. 1):307. (Abstr.)

16. Pongor, S., M. Brownlee, and A. Cerami. 1983. Preparation of high-potency, non-aggregating insulins using a novel sulfation procedure. Diabetes. 32:1087-1091.

17. Albisser, A. M., W. Lougheed, K. Perlman, and A. Bahoric. 1980. Nonaggregating insulin solutions for long-term glucose control in experimental and human diabetes. Diabetes. 29:241-243.

18. Schade, D. S., R. P. Eaton, J. DeLongo, L. C. Saland, A. J. Ladman, and G. A. Carlson. 1982. Electron microscopy of insulin preparations. Diabetes Care. 5:25-30.

19. Martindale, H., J. Marsh, F. R. Hallet, and A. M. Albisser. 1982. Examination of insulin formulations using quasi-elastic light scattering. Diabetes. 31:364-366.

20. Burke, M. J., and M. A. Rougvie. 1972. Cross-beta protein structures. I. Insulin fibrils. Biochemistry. 11:2435-2439.

21. Fisher, B. V., and P. B. Porter. 1981. Stability of bovine insulin. J. Pharm. Pharmacol. 33:203-206.

22. Sato, S., C. D. Ebert, and S. W. Kim. 1983. Prevention of insulin self-association and surface adsorption. J. Pharm. Sci. 72:228-232.

23. Smith, D. J., and R. M. Venable. 1985. Separation and quantitation of insulins and related substances in bulk insulin crystals and in injectables by reversed-phase high performance liquid chromatography and the effect of temperature on the separation. J: Chromatogr. Sci. 23 81-88.

24. Tatnell, M. A., R. H. Jones, and P. H. Sönksen. 1984. Covalentlylinked insulin dimers: their metabolism and biological effects in vivo as partial competitive antagonists of insulin clearance. Diabetologia. 27 27-31.

25. Hayford, J. T., and R. G. Thompson. 1982. Free and total insulin integrated concentrations in insulin-dependent diabetes. Metab. Clin. Exp. 31:387-397.

26. Schernthaner, G., M. Borkenstein, M. Fink, W. R. Mayr, J. Menzel, and E. Schober. 1983. Immunogenicity of human insulin (novo) or pork monocomponent insulin in HLA-DR-typed insulin-dependent diabetic individuals. Diabetes Care. 6(Suppl.)43:48.

27. Colnes, J. P., and P. M. Mead. 1985. Absolute and relative concentrations of circulating aggregates of insulin in IDDM as a function of insulin species and dose. Diabetes. 34(Suppl. 1):364. (Abstr.) 